\title{
Are there differences between de novo and secondary upper tract urothelial carcinoma tumours?
}

Hanan Goldberg ${ }^{1 *}$; Douglas C. Cheung ${ }^{1 *}$; Thenappan Chandrasekar ${ }^{1}$; Zachary Klaassen ${ }^{1}$; Christopher J.D. Wallis ${ }^{1}$; Girish S. Kulkarni ${ }^{1}$; Rashid Sayyid ${ }^{1}$; Andrew Evans ${ }^{2}$; Mehdi Masoomian²; Bharati Bapat $^{3}$; Theodorus van der Kwast ${ }^{2}$, Robert J. Hamilton ${ }^{1}$; Alexandre Zlotta $^{1}$; Neil Fleshner ${ }^{1}$

${ }^{1}$ Division of Urology, Department of Surgical Oncology, Princess Margaret Cancer Centre, University Health Network and the University of Toronto, Toronto, ON, Canada; ${ }^{2}$ Pathology Department, Princess Margaret Cancer Centre, University Health Network and the University of Toronto, Toronto, ON, Canada; ${ }^{3}$ Department of Laboratory Medicine and Pathobiology, University of Toronto, and Lunenfeld Tanenbaum Research Institute, Sinai Health System, Toronto, ON, Canada

${ }^{*}$ Equal contribution

Cite as: Can Urol Assoc J 2019 January 21; Epub ahead of print. http://dx.doi.org/10.5489/cuaj.5595

Published online January 21, 2019

$* * *$

\section{Abstract}

Introduction: Upper tract urothelial carcinoma (UTUC) accounts for $<5 \%$ of all urothelial cancers. We aimed to ascertain the clinical differences between UTUC tumours presenting de novo (DnUTUC) and those presenting secondary (SUTUC) following a bladder cancer diagnosis.

Methods: Our institutional database was queried for all UTUC patients who were surgically treated with radical nephroureterectomy or ureterectomy between 2003 and 2017. Bladder recurrence and cancer-specific mortality were compared. To reduce the possible bias due to confounding variables obtained from a simple comparison of outcomes, DnUTUC patients were matched (for age, gender, tumour location, type of surgery, grade, TNM staging, presence of carcinoma in situ, and lymphovascular invasion) with propensity score to SUTUC patients. Bladder recurrence and cancer-specific mortality were assessed with Cox proportional hazards model.

Results: A total of 117 UTUC patients were identified: 80 with DnUTUC (68.4\%) and 37 with SUTUC (31.6\%). A greater proportion of males with SUTUC was demonstrated (89.2\% vs. 68.8; $\mathrm{p}=0.02$ ). In both groups, $67.5 \%$ of patients had high-grade disease, but SUTUC demonstrated a higher carcinoma in situ rate (43.2\% vs. 25\%; $\mathrm{p}=0.047)$. Univariate analysis demonstrated that the five-year bladder recurrence rate was trending to be higher in SUTUC (65.3\% vs. 20.5\%; 
$\mathrm{p}=0.099)$. In the Cox model, however, it was associated with increased bladder recurrence (hazard ratio [HR] 3.69; 95\% confidence interval [CI] 1.68-8.09; p=0.001). Although univariate analysis demonstrated that SUTUC patients were more likely to die of their disease (30.6\% vs. 9\%; $\mathrm{p}=0.009$ ), the multivariable Cox model did not demonstrate this association. The limitations of this study include its retrospective, single-centre design and relatively small cohort of patients. Conclusions: In this hypothesis-generating study, some evidence suggests that further research is needed to delineate differences between SUTUC and DnUTUC.

\section{Introduction}

Urothelial carcinomas, which may occur anywhere along the urothelial epithelium from the lower (urethra and bladder) to the upper (ureter, renal pelvis, and calyces) urinary tract, together are categorized as the fifth most common tumour site. ${ }^{1}$. Upper tract urothelial cancer (UTUC), occurring in the ureter, renal pelvis or calyces, accounts for $5-10 \%$ of all urothelial carcinoma. ${ }^{2}$ These tumours are most common in people in their $7^{\text {th }}$ decade, and more common in men. ${ }^{3}$ Although also arising from urothelium, UTUC is increasingly being recognized as a different disease entity from bladder urothelial carcinoma. Firstly, although still controversial, evidence exists that bladder carcinoma occurs 3-4 times more commonly in men ${ }^{4}$ compared to the ratio of 2:1 for men in UTUC ${ }^{3,5}$. Secondly, it has inferior survival in women, while this is not observed in UTUC. ${ }^{6}$ Thirdly, at initial diagnosis, UTUC are more invasive tumours than bladder carcinoma (60\% vs. $15-25 \%){ }^{7,8}$ Lastly, recurrence in the form of UTUC is less likely to develop after bladder carcinoma (2-6\%) ${ }^{9}$ than for bladder carcinoma to develop after UTUC (22-47\%). ${ }^{10}$ Despite these clear differences, the underlying pathophysiology and management of UTUC have traditionally been extrapolated from our understanding and management of bladder carcinoma. ${ }^{11}$ However, there is increasing evidence to suggest that UTUC has unique epidemiologic, histologic, molecular, and prognostic factors compared to bladder carcinoma. ${ }^{12}$

This led us to hypothesize that primary de-novo UTUC (DnUTUC) is a distinct entity from secondary UTUC (SUTUC) which develops after a bladder carcinoma diagnosis and might me more closely associated with it. To date, no series has stratified UTUC in this manner, and studied the differences, if any, between DnUTUC and SUTUC.

\section{Methods}

\section{Patient cohort}

After receipt of approval by the institutional ethics committee, we performed a retrospective chart review of all patients older than 18 who underwent radical definitive surgery (nephroureterectomy or distal ureterectomy) for UTUC between 2003 and 2017, from the Princess Margaret Cancer Centre institutional database. We excluded all patients who presented with metastatic disease. Additionally, due to the inclusion criteria, UTUC patients who 
underwent conservative or endoscopic management were also excluded. Conservative management of UTUC does not commonly occur in our facility, and this type of treatment is usually undertaken at another facility. The purpose of these exclusions was to compare outcomes of UTUC patients with localized disease who were treated with a definitive radical treatment.

\section{Surgical procedure and follow-up protocol}

All nephroureterectomies were performed with excision of the entire ipsilateral ureter with a formal extra-vesical bladder cuff removal. All ureterectomy procedures were open procedures, while all nephroureterectomies were either open or laparoscopic combined with either a small open midline infra-umbilical or Gibson incision for removal of the distal ureter and bladder-cuff. None of the patients in this cohort received postoperative intravesical instillation of Mitomycin. Patients were stratified into two groups: those with DnUTUC and those with SUTUC.

Following surgery, patients who underwent either nephroureterectomy or ureterectomy were seen in the clinic every three months for the first year, and every six months from the second to the fifth year, and annually thereafter. In each clinic visit the following tests were performed, including a complete history, physical examination, blood tests (including complete blood count, and serum chemistry), urine cytology, cystoscopic examination of the bladder, and radiographic evaluation of the contralateral upper urinary tract either by CT scan or ultrasound. Bladder recurrences was defined as definitive cystoscopic evidence of a tumour. Cause of death was defined by chart review or by death certificates.

\section{Covariates and outcomes}

We collected data on relevant demographic and tumour data including patient age, gender, age adjusted Charlson comorbidity score, smoking history, history of bladder carcinoma, tumour laterality and location, type of surgery (nephroureterectomy or distal ureterectomy, open vs. laparoscopic), pathologic grade, TNM staging, presence of carcinoma in-situ (CIS), and lymphovascular invasion, and receipt of neoadjuvant and adjuvant chemotherapy. Outcomes data collected included bladder recurrence, follow-up duration, cancer specific mortality (CSM), and last known clinical status. Importantly, bladder recurrence was defined as development of bladder carcinoma postoperatively, at a time where the patient was considered to be with no evidence of disease.

\section{Statistical analysis}

Descriptive analyses (mean with standard deviation and median with interquartile range) was used for continuous variables, proportions for discrete variables, and comparative tests included chi-square for discrete variables and Kruskal-Wallis for continuous variables. Kaplan-Meier (KM) graphs (log rank test) was used to evaluate recurrence free survival (RFS), and cancer specific survival, stratified according to whether UTUC was de-novo or following a diagnosis of bladder carcinoma, and stratified according to the UTUC tumour location (renal pelvis or ureter). To reduce the possible bias due to confounding variables obtained from a simple comparison of 
outcomes, propensity score matching was performed. This was done (2:1 ratio) by age, gender, tumour location, type of surgery, tumour grade, TNM staging, presence of CIS and lymphovascular invasion. Cox proportional hazards regression model was performed to identify factors predicting bladder recurrence and CSM. A priori covariates in the model included type of UTUC (DnUTUC or SUTUC) and the calculated propensity score. Statistical tests were twotailed and a p-value $<0.05$ was considered statistically significant. All analyses were conducted using SPSS software version 23.0 (SPSS Inc., Chicago, IL) and SAS 9.4 (SAS Institute, Cary, North Carolina).

\section{Results}

A total of 122 patients with UTUC who were treated with either nephroureterectomy or ureterectomy were identified over this time. Only three patients were treated conservatively in an endoscopic manner and were referred to another center. Out of the 122 patients, five presented with metastatic disease and thus were excluded, leaving 117 patients with DnUTUC (80 [68.4\%]) and SUTUC (37 [31.6\%]). While all patients were diagnosed with UC, one patient from the DnUTUC group had micropapillary features on his final pathology (1.25\%). Five DnUTUC patients (4.3\%) were diagnosed with Lynch syndrome, an autosomal dominant genetic syndrome, harboring a high risk of colon cancer as well as other malignancies, including UTUC. Table 1 demonstrates patient clinical and operative data. No statistically significant differences were noted in the age, age-adjusted Charlson score, smoking history, tumour laterality, and type of surgery patients underwent in both groups. However, the proportion of males in the SUTUC group was significantly higher ( $89.2 \%$ vs. $68.8 \%$, $\mathrm{p}=0.02)$. In both groups approximately $2 / 3$ of the tumours were in the renal pelvis (63.75\% and 64.9\% in the DnUTUC and SUTUC groups, respectively). SUTUC tumours developed after a mean time (SD) of 44 months (44.97) from bladder tumour treatment. Roughly $2 / 3$ of the patients underwent an open procedure $(60 \%$ and $64.9 \%$ of DnUTUC and SUTUC, respectively). Five of the open cases began as laparoscopic cases that were eventually converted to an open procedure.

Table 2 demonstrates pathologic and follow-up data. Most patients in both groups had high-grade disease (67.5\% and 67.6\% in DnUTUC and SUTUC, respectively), and T stage distribution were similar as well, with $47.5 \%$ of DnUTUC and $51.4 \%$ of SUTUC patients having pTa disease. Lymph node dissection was done in only a third of the patients in both groups (32.5\% and 32.4\%), and no difference could be seen in the pathologic node stage. Lymphovascular invasion and positive ureteral margin rates were also similar among both groups. CIS however, was significantly more common in the SUTUC group (43.2\% vs. 25\%, $\mathrm{p}=0.047$ ). Receipt of neoadjuvant and adjuvant chemotherapy, although similar in both groups, was not very common, with $3.75 \%$ and $5.4 \%$ of DnUTUC and SUTUC patients, respectively, receiving neoadjuvant chemotherapy, and $12.5 \%$ and $8.1 \%$ of DnUTUC and SUTUC patients, respectively, receiving adjuvant chemotherapy. 
Median follow-up was similar with 32.5 months (IQR 11.4-61.4) in DnUTUC and 39.5 months IQR (20.6-63.4) in SUTUC, p=0.22. A total of 10 (of 37, 27\%) SUTUC patients had undergone radical cystectomy prior to the development of SUTUC. After excluding these patients, although not statistically significant, the five-year bladder recurrence rate was demonstrated to be higher in the SUTC group (65.3\% vs. 20.5\%, p=0.099), as seen in the KM analysis (Figure 1a). However, there was a statistical significant difference in bladder recurrence rates after stratifying by tumour location for DnUTUC only tumours (Figure 1b), demonstrating renal pelvis tumours to have a lower five-year recurrence rate of $12.2 \%$ vs. $34.5 \%, p=0.047$ ). No such difference was noted for the SUTUC patients (Figure 1c), with five-year bladder recurrence rate of $66.6 \%$ vs. $62.5 \%$, for renal pelvis and ureteral tumours, respectively, $\mathrm{p}=0.242$.

Overall 15/37 (40.5\%) SUTUC patients underwent RC, 10 patients prior to the development of SUTUC, and 5 patients following SUTUC diagnosis (18.5\%). In contrast only 2/80 DnUTUC patients (2.5\%) developed bladder carcinoma and underwent radical cystectomy following DnUTUC diagnosis.

Propensity score matching with a 2:1 ratio was performed and resulted in a total of 83 patients being matched (54 in the DnUTUC group and 29 in the SUTUC group, supplemental table 1). Cox proportional hazards regression analysis using the propensity score and type of UTUC (DnUTUC or SUTUC) evaluated the predictors of bladder recurrence (after excluding SUTUC patients who underwent cystectomy prior to SUTUC development), as seen in table 3. This model demonstrated that SUTUC compared to DnUTUC was strongly associated with increased bladder recurrence rate (HR 3.837, 95\% C.I. 1.689-8.091, p=0.001).

CSM was significantly worse in SUTUC patients as shown in the KM curve in figure 2a (five-year CSM rate of 22\% vs. 9\%, log rank $\mathrm{p}=0.011$ ). However, for both groups, no difference was discerned between CSM in renal pelvic and ureteral tumours $(10.2 \%$ vs. $7.1 \%, \mathrm{p}=0.121$, and $21 \%$ vs. $25 \%$, p=0.714 for DnUTUC and SUTUC, respectively), as can be seen in figure $2 \mathrm{~b}$ and 2c. However, the Cox proportional hazards model demonstrated that although SUTUC was associated with worse CSM, this was not statistically significant (HR 2.246, 95\% C.I. 0.796.391) (Table 3). A total of 11 (30.6\%) SUTUC patients compared to only 7 (9\%) DnUTUC patients, died of urothelial carcinoma during the study follow-up period $(\mathrm{p}=0.009)$.

\section{Discussion}

In this cohort, most patients diagnosed with UTUC had de-novo disease, without a prior history of bladder carcinoma. Pathologic characteristics, including primary tumour stage, grade and lymph-node involvement were similar between patients with DnUTUC and SUTUC. No clear pathologic disparities could be discerned between DnUTUC and SUTUC aside from the higher rate of CIS in SUTUC patients. Although not statistically significant, there was a trend showing that the five-year bladder recurrence rate was higher in the SUTUC group (65.3\% vs. 20.5\%, $\mathrm{p}=0.099)$. Moreover, the five-year CSM rate was significantly worse for SUTUC patients. However, in Cox proportional hazards regression analysis, adjusting for some of the relevant 
covariates, through the usage of the propensity score, SUTUC was demonstrated to be a significant predictor of bladder recurrence, but not of CSM. An important difference demonstrated between the groups was the fact that five DnUTUC patients (4.3\%) were diagnosed with Lynch syndrome, an autosomal dominant genetic syndrome, harboring a high risk of colon cancer and other malignancies, including UTUC. This interesting genetic difference between the groups, supports the hypothesis that DnUTUC is a distinct entity when compared to SUTUC, that might be more closely related to bladder cancer.

Reconciling whether SUTUC tumours are simply primary bladder recurrences or new primary tumours is a controversial and daunting task. It is known that urothelial carcinoma is a multifocal disease, with at least $30 \%$ of patients presenting with multifocal tumours. ${ }^{13}$ It has also been shown that abnormalities of the surrounding urothelium are found near the base of superficial bladder tumours in $25 \%$ of cases ${ }^{14}$, which are intimately associated with recurrence and invasion. ${ }^{15}$ In the past, these clinical observations led to the formation of the "field change" concept. ${ }^{16}$ According to this concept, exposure to carcinogens results in the independent transformation of epithelial cells to a preconditioned epithelium. This eventually results in the creation of multifocal or metachronous, genetically independent, tumours. ${ }^{16}$ However, this concept is less accepted today and has been mostly replaced by the theory of clonal origin of multiple urothelial carcinomas. ${ }^{17}$ This theory hypothesized that the progeny of a single transformed cell spreads through the urothelium resulting in topographically and chronologically distinct, but genetically related tumours. Therefore, the often observed multifocality of urothelial carcinomas is a result from intraluminal migration and re-implantation. When the primary tumour initially manifests in the urothelium, cells from which future tumours might arise, may already be present, distal from it, along the urothelium. The fact that patients with UTUC have a $22-47 \%$ risk of bladder tumours, but only a 2-3\% risk of contralateral UTUC, and that UTUC develops in only $2-6 \%$ of patients with bladder carcinoma ${ }^{6}$ favors the clonality concept. If this theory is correct, SUTUC tumours, should be prescribed as new primary tumours. ${ }^{18}$ However, it is also very likely that these SUTUC tumours originated from the primary bladder tumour (possibly resulting from intra-epithelial migration) and, therefore, the SUTUC tumour may be a recurrence of bladder carcinoma. Furthermore, in the SUTUC patients, it is impossible to delineate whether future bladder recurrences originated from the original bladder tumour or from the SUTUC itself. In any case, due to the inconclusive findings in the literature and until more data is discovered, it is still unclear whether SUTUC is labeled as a recurrence or as a primary tumour. $^{18}$

Our study demonstrates that at least pathologically, there was no difference between DnUTUC and SUTUC, except, the higher rate of CIS, which could favor the explanation that SUTUC is an extension of bladder carcinoma or represents a recurrence of it. The simple univariate analysis demonstrates a worse CSM rate in SUTUC patients, although this correlation disappeared in the multivariable analysis. Furthermore, although univariate analysis did not 
demonstrate a statistically significant higher bladder recurrence rate among SUTUC patients, it was seen in the multivariable analysis. Although our goal was to compare UTUC patients with and without bladder cancer history, it was impossible for the propensity score to control for the bladder cancer history. However, the variable of whether the disease was SUTUC or DnUTUC was included in the multivariable model to try compare between both groups. The multivariable analyses demonstrated that SUTUC was only a predictor of bladder recurrence, which might well be explained by the thought that SUTUC is in fact a bladder recurrence, and not a separate disease entity of the upper tract. This study does, to our opinion, suggests that there is further room to explore differences between UTUC with and without a history of bladder cancer. Deciphering the true origin of SUTUC will most likely be achieved with utilization of tumour specific genetic mutations, as has been done to differentiate between muscle invasive and nonmuscle invasive bladder tumours, ${ }^{19,} 20$ and between UTUC and bladder carcinoma. ${ }^{4}$ Most recently there have been published data demonstrating UTUC specific genetic alterations. ${ }^{21}$ Applying the methods used in that study could help differentiate between DnUTUC and SUTUC and at least partly answer our questions.

The proportion of DnUTUC patients, tumour location, mean age, and pathologic parameters in our cohort were similar to those reported by Cha et al. in their multi-institutional study including more than 2200 patients. $^{22}$ Our study exhibited a similar rate of chemotherapy usage to that shown in large population based studies. Such a study, using the Surveillance, Epidemiology and End Results (SEER)-Medicare registry between 2002-2011, demonstrated utilization of neoadjuvant and adjuvant chemotherapy in $1.8 \%$ and $11.8 \%$ of cases, respectively. ${ }^{23}$ This is slightly lower than our neoadjuvant rate (3.75-5.4\%) but coinciding with our adjuvant rate (8.1-12.5\%). Our lymph node dissection rate is quite higher than that reported in other studies. Pearce et al. reported a rate of $15 \%$ in a large population based study ${ }^{24}$, considerably lower than our 32.5\% rate. Usage of laparoscopy in approximately $38 \%$ of our cohort is also similar that reported in previous large studies. ${ }^{25}$

It has been shown that patients who underwent RC, are at increased risk of UTUC recurrence. ${ }^{26}$ There is no doubt that the fact that in the SUTUC group 15 patients $(40.6 \%)$ had undergone RC, compared to only 2 patients (2.5\%) in the DnUTUC group, had a significant effect on their CSM rates, although we did try to account for that difference, by excluding from our analyses all patients who underwent a radical cystectomy prior to SUTC development.

Ureteral tumour location was demonstrated to predict higher bladder recurrence rates in the DnUTUC group in our cohort. The literature assessing the impact of UTUC tumour location on its outcomes is conflicting. There are several studies showing that ureteral tumours are correlated with recurrence, ${ }^{27,} 28$ as shown in our DnUTUC group. In contrast, there are a number of studies demonstrating no difference in recurrence rates between UTUC tumours arising from both the renal pelvis and ureter, ${ }^{29,30}$ as shown in our SUTUC group. 
Our study has several limitations, most notably its retrospective nature and relatively small sample size originating from a single center. The inherent problem of delineating whether SUTUC is a new primary or a bladder tumour recurrence, and the true origin of the ensuing recurrences, whether from the original bladder tumour or from the upper tract, might possibly have caused this group to be contaminated. Adding to this point, it is important to note that in all the KM curves comparing DnUTUC and SUTUC, there is an inherent immortal time bias for the SUTUC patients, as the comparison did not consider the time from bladder carcinoma diagnosis to SUTUC diagnosis. This is especially problematic if the bladder recurrence and CSM reported in our study originated from their primary bladder tumour, and not from their SUTUC tumour. Additionally, we lacked data on tumour size, tumour architecture (sessile or papillary), and tumour multifocality, which could have been an important parameter, as multifocality, and sessile UTUC tumours have been shown to predict recurrence. ${ }^{22}$ Lastly, our follow-up period of approximately 3 years, was relatively short and this prevented us to appreciate bladder recurrence and CSM outcomes over a long course. However, this is a hypothesis generating study, and the largest study to date to identify and risk stratify UTUC patients according to DnUTUC and SUTUC type.

\section{Conclusion}

The data from our study does suggest that there is further space to explore differences between DnUTUC and SUTUC. It is still unclear whether SUTUC represents a worse disease, however, we strongly support the European guidelines in adhering to the strict follow-up strategy following surgery, of frequent cystoscopies and upper-tract imaging in all patients, whether DnUTUC or SUTUC ${ }^{6}$. Whether a difference exists between these two entities, leading to a specific and unique follow-up protocol for each disease type, remains to be discovered. 


\section{References}

1. Siegel RL, Miller KD, Jemal A. Cancer statistics, 2016. CA: a cancer journal for clinicians 2016; 66(1): 7-30.

2. Munoz JJ, Ellison LM. Upper tract urothelial neoplasms: incidence and survival during the last 2 decades. The Journal of urology 2000; 164(5): 1523-5.

3. Shariat SF, Favaretto RL, Gupta A, et al. Gender differences in radical nephroureterectomy for upper tract urothelial carcinoma. World journal of urology 2011; 29(4): 481-6.

4. Fajkovic H, Halpern JA, Cha EK, et al. Impact of gender on bladder cancer incidence, staging, and prognosis. World journal of urology 2011; 29(4): 457-63.

5. Lughezzani G, Sun M, Perrotte P, et al. Gender-related differences in patients with stage I to III upper tract urothelial carcinoma: results from the Surveillance, Epidemiology, and End Results database. Urology 2010; 75(2): 321-7.

6. Roupret M, Babjuk M, Comperat E, et al. European Association of Urology Guidelines on Upper Urinary Tract Urothelial Carcinoma: 2017 Update. European urology 2018; 73(1): 111-22.

7. Margulis V, Shariat SF, Matin SF, et al. Outcomes of radical nephroureterectomy: a series from the Upper Tract Urothelial Carcinoma Collaboration. Cancer 2009; 115(6): 1224-33.

8. Babjuk M, Bohle A, Burger M, et al. EAU Guidelines on Non-Muscle-invasive Urothelial Carcinoma of the Bladder: Update 2016. European urology 2017; 71(3): 44761.

9. Li WM, Shen JT, Li CC, et al. Oncologic outcomes following three different approaches to the distal ureter and bladder cuff in nephroureterectomy for primary upper urinary tract urothelial carcinoma. European urology 2010; 57(6): 963-9.

10. Seisen T, Granger B, Colin P, et al. A Systematic Review and Meta-analysis of Clinicopathologic Factors Linked to Intravesical Recurrence After Radical Nephroureterectomy to Treat Upper Tract Urothelial Carcinoma. European urology 2015; 67(6): 1122-33.

11. Mathieu R, Bensalah K, Lucca I, Mbeutcha A, Roupret M, Shariat SF. Upper urinary tract disease: what we know today and unmet needs. Translational andrology and urology 2015; 4(3): 261-72.

12. Green DA, Rink M, Xylinas E, et al. Urothelial carcinoma of the bladder and the upper tract: disparate twins. The Journal of urology 2013; 189(4): 1214-21.

13. Kiemeney LA, Witjes JA, Verbeek AL, Heijbroek RP, Debruyne FM. The clinical epidemiology of superficial bladder cancer. Dutch South-East Cooperative Urological Group. British journal of cancer 1993; 67(4): 806-12.

14. Wolf H, Hojgaard K. Urothelial dysplasia in random mucosal biopsies from patients with bladder tumours. Scandinavian journal of urology and nephrology 1980; 14(1): 37-41.

15. Wolf H, Hojgaard K. Urothelial dysplasia concomitant with bladder tumours as a determinant factor for future new occurrences. Lancet (London, England) 1983; 2(8342): 134-6. 
16. Slaughter DP, Southwick HW, Smejkal W. Field cancerization in oral stratified squamous epithelium; clinical implications of multicentric origin. Cancer 1953; 6(5): 963-8.

17. Sidransky D, Frost P, Von Eschenbach A, Oyasu R, Preisinger AC, Vogelstein B. Clonal origin of bladder cancer. The New England journal of medicine 1992; 326(11): 737-40.

18. Aben KK, Witjes JA, van Dijck JA, Schalken JA, Verbeek AL, Kiemeney LA. Lower incidence of urothelial cell carcinoma due to the concept of a clonal origin. European journal of cancer (Oxford, England : 1990) 2000; 36(18): 2385-9.

19. Weinstein JN AR, Broom BM, Wang W, et al. Comprehensive molecular characterization of urothelial bladder carcinoma. Nature 2014; 507(7492): 315-22.

20. Zhao YG, Shi BY, Qian YY, Bai HW, Xiao L, He XY. Dynamic expression changes between non-muscle-invasive bladder cancer and muscle-invasive bladder cancer. Tumouri 2014; 100(6): e273-81.

21. Moss TJ, Qi Y, Xi L, et al. Comprehensive Genomic Characterization of Upper Tract Urothelial Carcinoma. European urology 2017; 72(4): 641-9.

22. Cha EK, Shariat SF, Kormaksson M, et al. Predicting clinical outcomes after radical nephroureterectomy for upper tract urothelial carcinoma. European urology 2012; 61(4): 818-25.

23. Cohen A, Kuchta K, Park S. Neoadjuvant and adjuvant chemotherapy use in upper tract urothelial carcinoma. Urologic oncology 2017; 35(6): 322-7.

24. Pearce SM, Pariser JJ, Patel SG, Steinberg GD, Shalhav AL, Smith ND. The effect of surgical approach on performance of lymphadenectomy and perioperative morbidity for radical nephroureterectomy. Urologic oncology 2016; 34(3): 121.e15-21.

25. Huang WW, Huang HY, Liao AC, et al. Primary urothelial carcinoma of the upper tract: important clinicopathological factors predicting bladder recurrence after surgical resection. Pathology international 2009; 59(9): 642-9.

26. Sanderson KM, Cai J, Miranda G, Skinner DG, Stein JP. Upper tract urothelial recurrence following radical cystectomy for transitional cell carcinoma of the bladder: an analysis of 1,069 patients with 10-year followup. The Journal of urology 2007; 177(6): 2088-94.

27. Akdogan B, Dogan HS, Eskicorapci SY, Sahin A, Erkan I, Ozen H. Prognostic significance of bladder tumour history and tumour location in upper tract transitional cell carcinoma. The Journal of urology 2006; 176(1): 48-52.

28. Park S, Hong B, Kim CS, Ahn H. The impact of tumour location on prognosis of transitional cell carcinoma of the upper urinary tract. The Journal of urology 2004; 171(2 Pt 1): 621-5.

29. Hall MC, Womack S, Sagalowsky AI, Carmody T, Erickstad MD, Roehrborn CG. Prognostic factors, recurrence, and survival in transitional cell carcinoma of the upper urinary tract: a 30-year experience in 252 patients. Urology 1998; 52(4): 594-601.

30. Raman JD, Ng CK, Scherr DS, et al. Impact of tumour location on prognosis for patients with upper tract urothelial carcinoma managed by radical nephroureterectomy. European urology 2010; 57(6): 1072-9. 


\section{Figures and Tables}

Fig. 1. Bladder recurrence-free survival in (a) all patients; (b) de novo upper tract urothelial carcinoma (UTUC) patients; and (c) secondary UTUC patients.
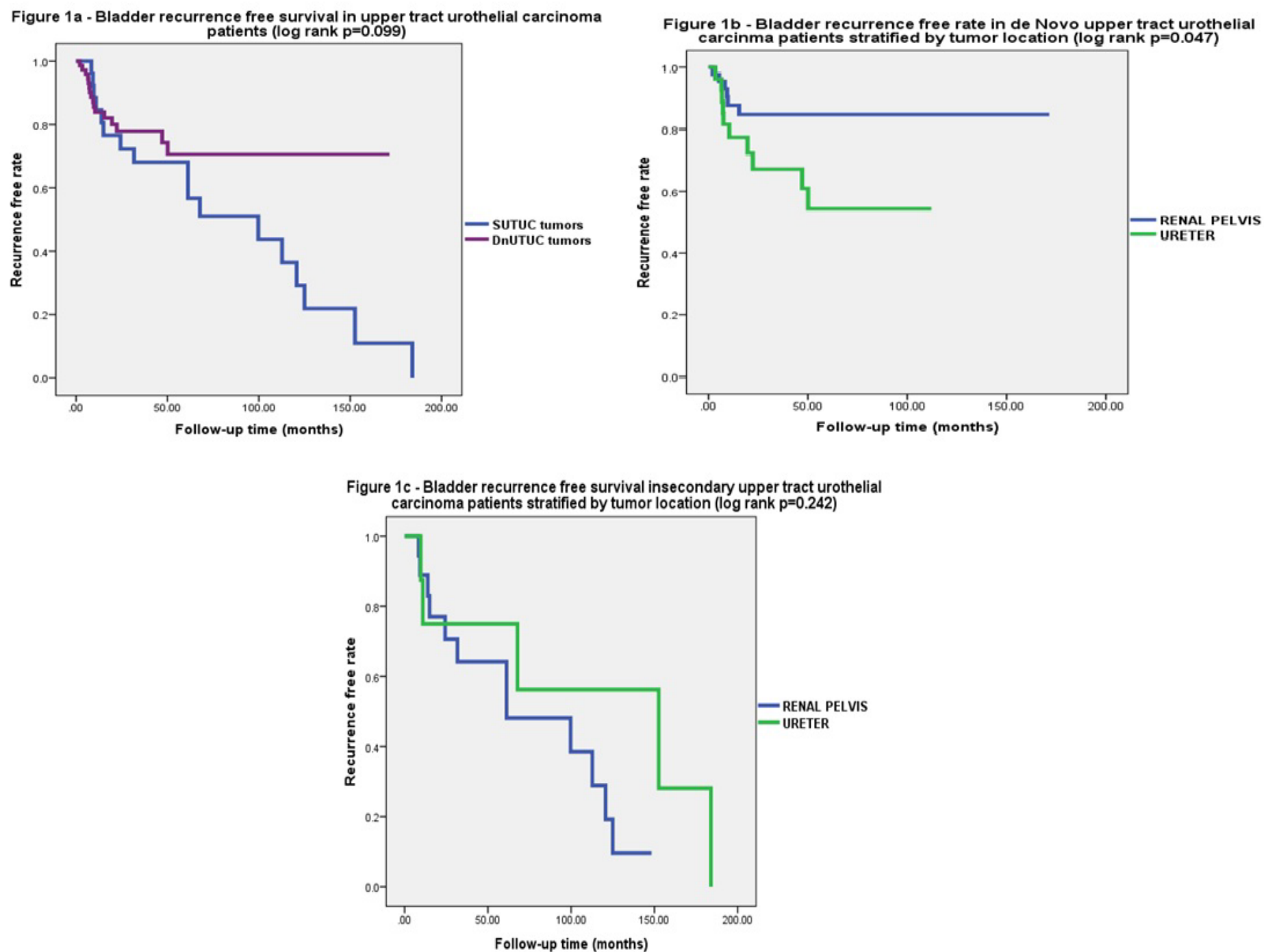
Fig. 2. Cancer-specific survival in (a) all patients; (b) de novo upper tract urothelial carcinoma (UTUC) patients; and (c) secondary UTUC patients.
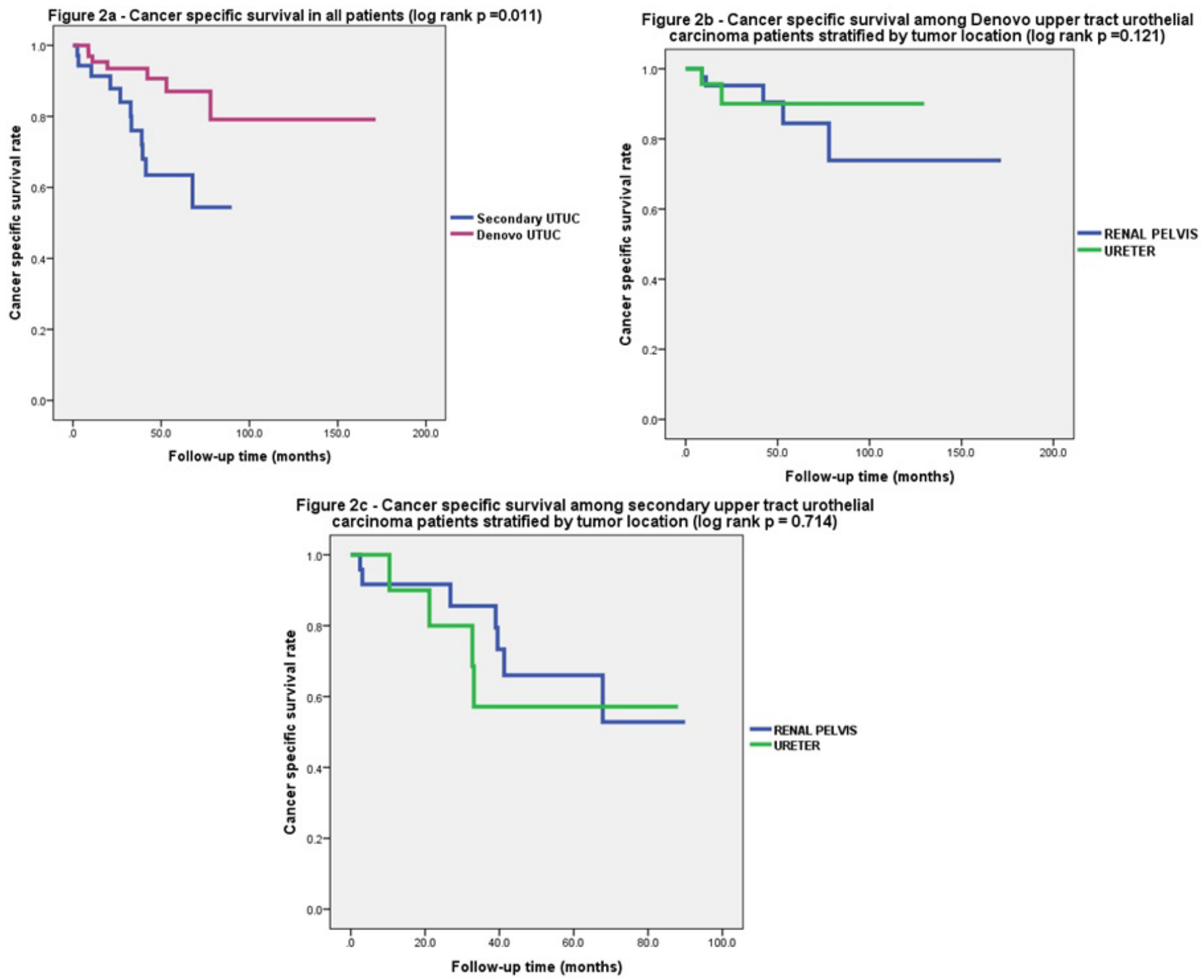


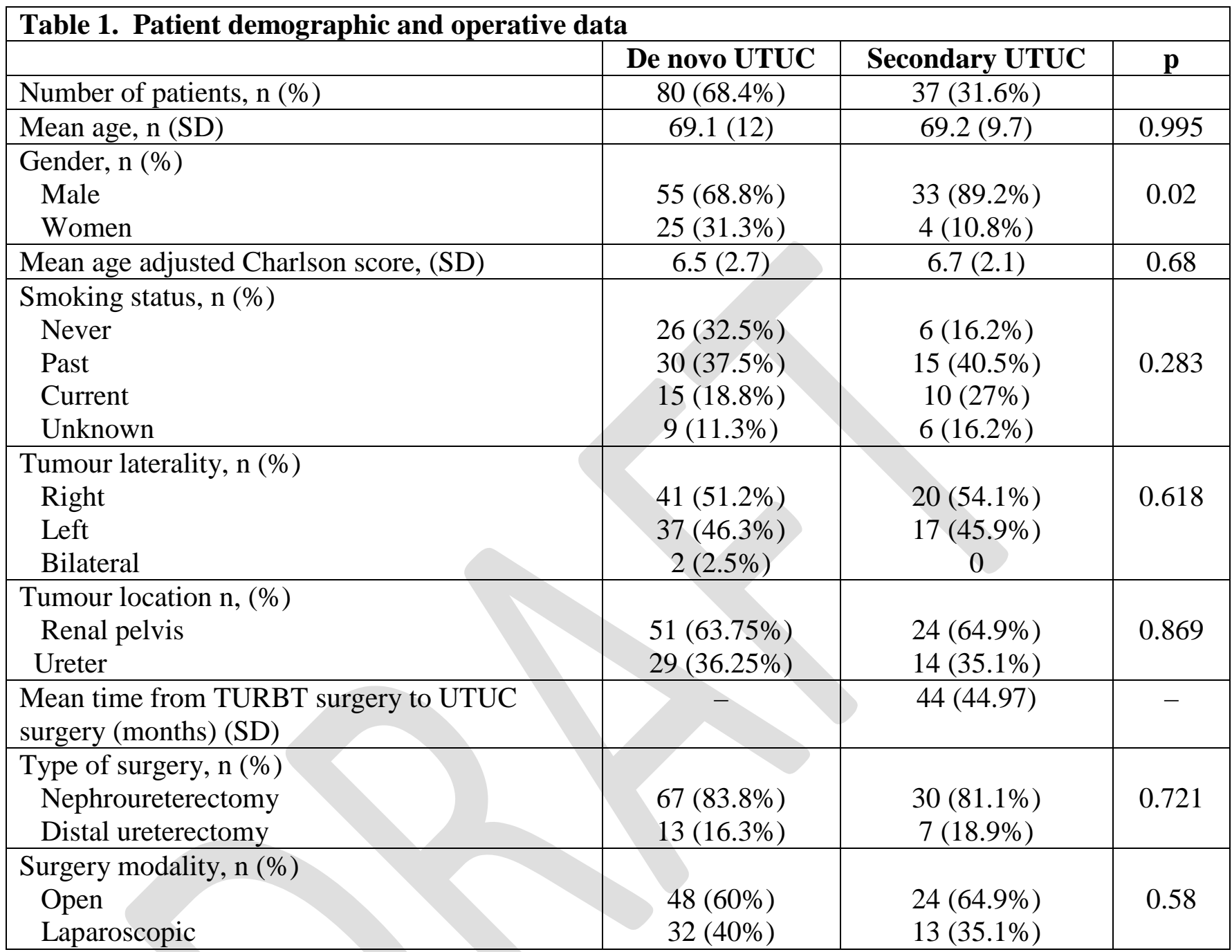

SD: standard deviation; TURBT: transurethral resection of bladder tumour; UTUC: upper tract urothelial carcinoma. 


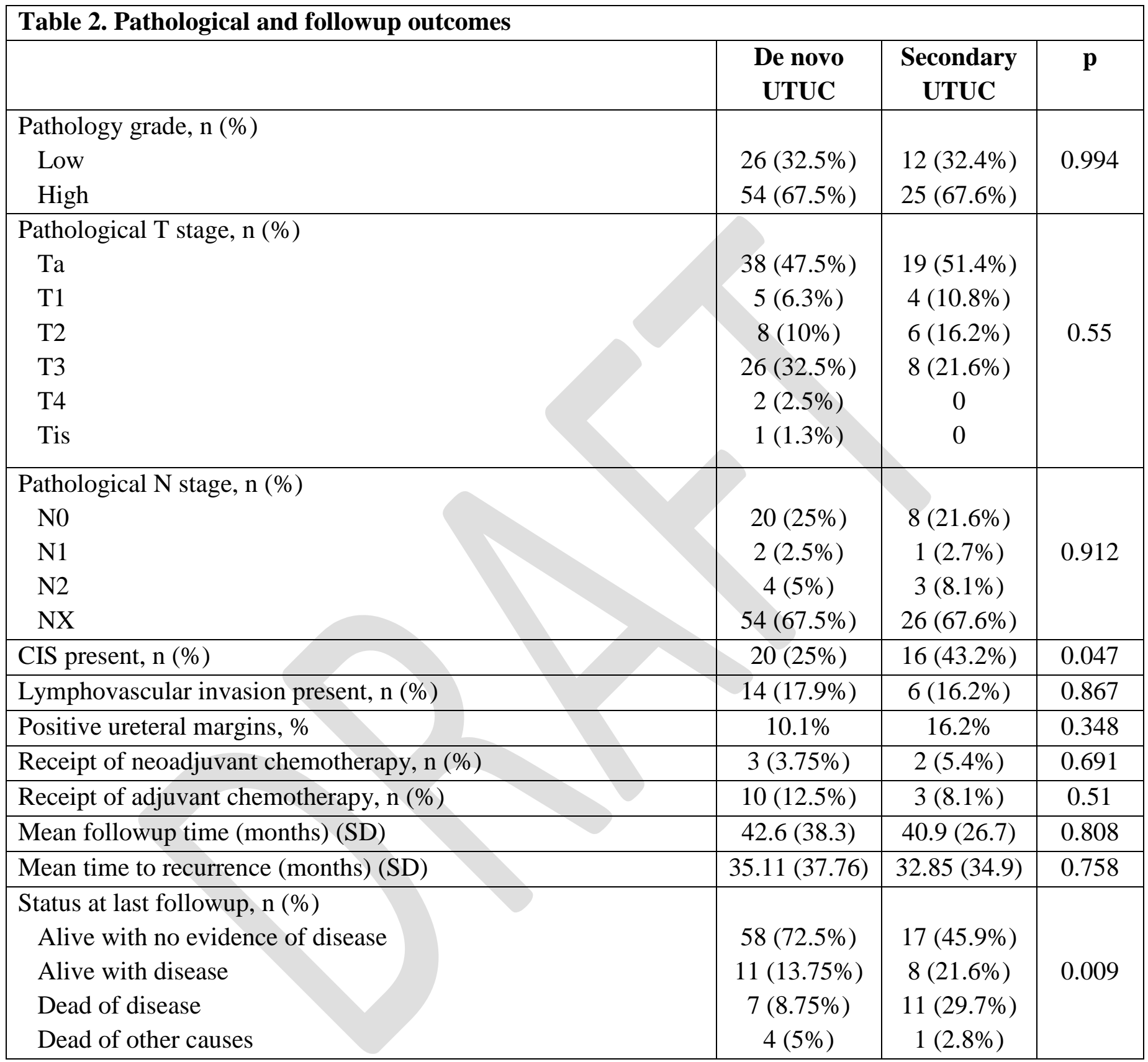

CIS: carcinoma in situ; SD: standard deviation; UTUC: upper tract urothelial carcinoma. 


\begin{tabular}{|c|c|c|c|c|c|c|c|c|}
\hline & \multicolumn{4}{|c|}{ Bladder recurrence } & \multicolumn{4}{|c|}{ Cancer-specific mortality } \\
\hline & \multirow{2}{*}{ HR } & \multicolumn{2}{|c|}{ 95\% CI for OR } & \multirow[b]{2}{*}{$\mathbf{p}$} & \multirow[t]{2}{*}{ HR } & \multicolumn{2}{|c|}{ 95\% CI for OR } & \multirow[b]{2}{*}{$\mathbf{p}$} \\
\hline & & Lower & Upper & & & Lower & Upper & \\
\hline $\begin{array}{l}\text { Type of UTUC (SUTUC vs. } \\
\text { DnUTUC) }\end{array}$ & 3.697 & 1.689 & 8.091 & 0.001 & 2.246 & 0.79 & 6.391 & 0.129 \\
\hline $\begin{array}{l}\text { Matched propensity score } \\
\text { (1:2 ratio) with match } \\
\text { criteria including age, } \\
\text { gender, tumour location, } \\
\text { type of surgery, tumour } \\
\text { grade, pT stage, pN stage, } \\
\text { CIS presence and LVI } \\
\text { presence }\end{array}$ & 3.837 & 0.971 & 15.165 & 0.055 & 3.63 & 0.563 & 23.388 & 0.175 \\
\hline
\end{tabular}

CI: confidence interval; CIS; carcinoma in situ; DnUTUC: de novo upper tract urothelial carcinoma; HR: hazard ratio; LVI: lymphovascular invasion; OR: odds ratio; SUTUC: secondary upper tract urothelial carcinoma. 


\begin{tabular}{|c|c|c|c|}
\hline \multicolumn{4}{|c|}{ Supplementary Table 1. Clinical parameters after propensity score matching (2:1 ratio) } \\
\hline & De novo UTUC & Secondary UTUC & $\mathbf{p}$ \\
\hline Number of patients, $\mathrm{n}(\%)$ & $54(65 \%)$ & $29(35 \%)$ & \\
\hline Mean age, $\mathrm{n}(\mathrm{SD})$ & $68.8(12.5)$ & $67.6(9.6)$ & 0.652 \\
\hline Gender, n (\%) & & & \\
\hline Male & $45(83.3 \%)$ & $25(86.2 \%)$ & 0.731 \\
\hline Women & $9(16.7 \%)$ & $4(13.8 \%)$ & \\
\hline Tumour location n, (\%) & & & \\
\hline Renal pelvis & $34(63 \%)$ & $19(65.5 \%)$ & 0.817 \\
\hline Ureter & $20(37 \%)$ & $10(34.5 \%)$ & \\
\hline Type of surgery, n (\%) & 2 & & \\
\hline Nephroureterectomy & $42(77.8 \%)$ & $23(79.3 \%)$ & 0.872 \\
\hline Distal ureterectomy & $12(22.2 \%)$ & $6(20.7 \%)$ & \\
\hline Pathology grade, n (\%) & & & \\
\hline Low & $20(37 \%)$ & $12(41.4 \%)$ & 0.698 \\
\hline High & $34(63 \%)$ & 17 (58.6\%) & \\
\hline Pathological T stage, n (\%) & & & \\
\hline $\mathrm{Ta}$ & $31(57.4 \%)$ & $16(55.2 \%)$ & \\
\hline $\mathrm{T} 1$ & $5(9.3 \%)$ & $3(10.3 \%)$ & 0.96 \\
\hline $\mathrm{T} 2$ & $5(9.3 \%)$ & 3 (10.3\%) & \\
\hline Т3 & $12(22.2 \%)$ & 7 (24.1\%) & \\
\hline $\mathrm{T} 4$ & $1(1.9 \%)$ & 0 & \\
\hline Pathological N stage, n (\%) & & & \\
\hline No & $11(20.4 \%)$ & $5(17.2 \%)$ & \\
\hline N1 & 0 & $1(3.4 \%)$ & 0.536 \\
\hline N2 & $4(7.4 \%)$ & $3(10.3 \%)$ & \\
\hline $\mathrm{NX}$ & $39(72.2 \%)$ & $20(69 \%)$ & \\
\hline CIS present, n (\%) & $13(24.1 \%)$ & $8(27.6 \%)$ & 0.726 \\
\hline Lymphovascular invasion present, n (\%) & $8(14.8 \%)$ & $5(17.2 \%)$ & 0.772 \\
\hline
\end{tabular}

CIS: carcinoma in situ; SD: standard deviation; UTUC: upper tract urothelial carcinoma. 